\title{
THE LASER DRIVEN ELECTRON ACCELERATOR EXPERIMENT AT STANFORD UNIVERSITY
}

\author{
R.L. Byer, T.Plettner ${ }^{\dagger}$, Y.C. Huang ${ }^{\ddagger}$, Ginzton Laboratories, Stanford University \\ E. Colby, R.L. Siemann, J.E. Spencer, H. Wiedemann, C. Barnes, SLAC \\ T.I. Smith, R.L. Swent, HEPL, Stanford University
}

\begin{abstract}
This experiment seeks to demonstrate laser driven particle acceleration in a dielectric loaded vacuum structure. Evidence for particle acceleration will be inferred from the change in the observed energy spread of the electron bunch. Under optimum conditions we expect an increase by a factor bigger than 3 . However, the energy profile as a function of several controllable beam parameters provides the most distinctive signature.
\end{abstract}

\section{INTRODUCTION}

The main motivation for considering an eventual accelerator built from a dielectric material and powered by a laser beam is the increase of the damage threshold of the structure for ultra short laser pulses [1], [2], and hence the achievable gradient of the cell by about an order of magnitude. The principle of acceleration from a pair of linearly crossed laser beams has been studied extensively in the past [3], and dielectric structures capable of 1 $\mathrm{GeV} / \mathrm{m}$ average gradient have been proposed [4], [5]. This is much lower than the peak gradient observed in plasma accelerators. However the main advantage of the crossed laser beam acceleration scheme is the absence of any material in the path of the particle beam. This allows, at least in principle, for the preservation of a low emittance and low energy spread of the particle beam and hence for indefinite cascading. Finally, a cleverly designed dielectric accelerator structure could allow for recycling of the laser beam, and therefore a very energy efficient system.

\section{THE EXPERIMENT}

The electron beam and laser beam parameters employed in this experiment are summarised in Table 1 . There are two important issues that determine the design and the kind of results expected in the experiment. One is the enormous mismatch in the repetition rate of the laser beam and the electron micro bunch train. Only one in ten thousand micro bunches may overlap with the laser pulse. In order to avoid the background from unusable micro bunches a fast kicker located in the main line deflects only

\footnotetext{
${ }^{*}$ Work supported by DOE contract DE-FG-97ER41043

† Email: tplettne@leland.stanford.edu

Fresently at the Department of Nuclear Science, National Tsing-Hua University, Hsinchu, Taiwan 30043
}

the desired micro bunch into a separate beam line where the experiment is located. The other important issue is the time duration of the e-beam pulse, which covers several optical cycles of the laser pulse. Therefore instead of observing an overall shift in the energy a change in the energy spread of the beam is expected. A high-resolution spectrometer magnet located downstream from the test laser accelerator cell measures the energy spread of the electron beam.

Table 1: Electron and laser beam parameters

\begin{tabular}{|c|c|c|}
\hline \multirow{4}{*}{$\begin{array}{c}\text { Ti:Sa } \\
\text { amplified } \\
\text { laser }\end{array}$} & Wavelength & $800 \mathrm{~nm}$ \\
\cline { 2 - 3 } & Pulse duration & $0.1-30 \mathrm{psec}$ \\
\cline { 2 - 3 } & Repetition rate & $10 \mathrm{~Hz}$ \\
\cline { 2 - 3 } & Energy per pulse & $0.055 \mathrm{~mJ}$ \\
\cline { 2 - 3 } & FWHM spot size & $130 \mu \mathrm{m}$ \\
\hline \multirow{4}{*}{$\begin{array}{c}\text { SCA } \\
\text { electron } \\
\text { beam }\end{array}$} & Energy & $32 \mathrm{MeV}$ \\
\cline { 2 - 3 } & Micropulse period & $85 \mathrm{nsec}$ \\
\cline { 2 - 3 } & Macropulse period & $0.1 \mathrm{sec}$ \\
\cline { 2 - 3 } & FWHMnch length & $2 \mathrm{psec}$ \\
\cline { 2 - 3 } & Electrons pergy bunch & $16 \mathrm{keV}$ \\
\cline { 2 - 3 } & Invariant emittance & $8 \pi \mathrm{mm}-\mathrm{mrad}$ \\
\hline
\end{tabular}

The expected effect is of the order of tens of $\mathrm{keV}$, therefore the key for the success of this experiment is the very low energy spread attainable at the SCA-FEL facility.

\subsection{The accelerator cell}

The accelerator cell consists of high reflector coated dielectric optics which are arranged such that the electron beam enters and leaves the cell through a vertical slit formed by these optics. The optics are mounted on motorised translation stages so that the size of the slits can be changed. A wide slit allows the electron beam to traverse the cell with no loss but truncates the laser field and severely reduces the energy gain. With a $5 \mu \mathrm{m}$ slit and a 1 psec laser pulse the energy spread of the electron beam is expected to increase by a factor of more than 3 , whereas a $100 \mu \mathrm{m}$ slit has virtually no effect. So far we have succeeded in sending the electron beam through a 50-60 $\mu \mathrm{m}$ slit with usable intensity, but have not been able to close the slits below $30 \mu \mathrm{m}$ and still observe the beam. The detailed geometry of the accelerator cell has been 
described in detail in previous papers [6]. A simplified diagram of the accelerator cell is given in Fig. 1.

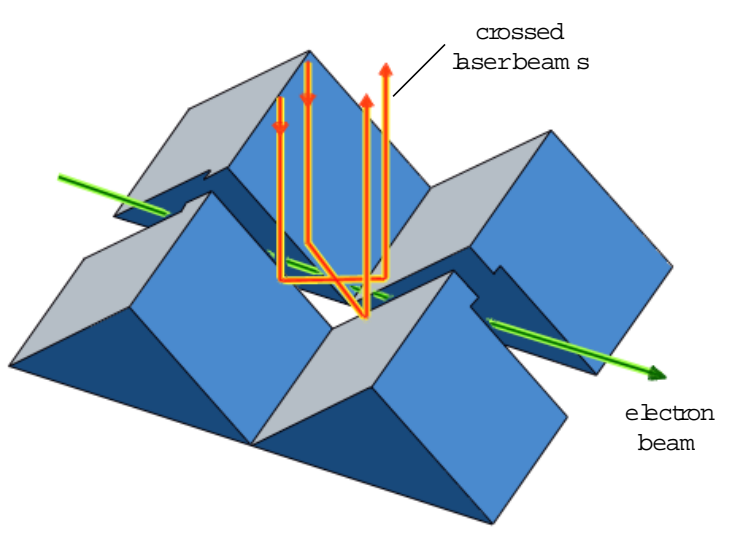

Figure 1: The accelerator cell.

\subsection{Timing of the laser and the electron beam}

The two beams have to be timed to within 1 psec in order to achieve good temporal overlap. Two monitors are employed; one coarse timing monitor that can detect the timing difference of the two beams to within $1 \mathrm{nsec}$, and a fine timing monitor capable of measuring timing changes between the beams with a 1 psec resolution. The coarse timing monitor is a silicon photodiode detector placed at the straight ahead port of the spectrometer that is capable of observing both beams. The fine timing system compares the timing between the modelocked laser, whose amplified pulse is sent to the accelerator, with the

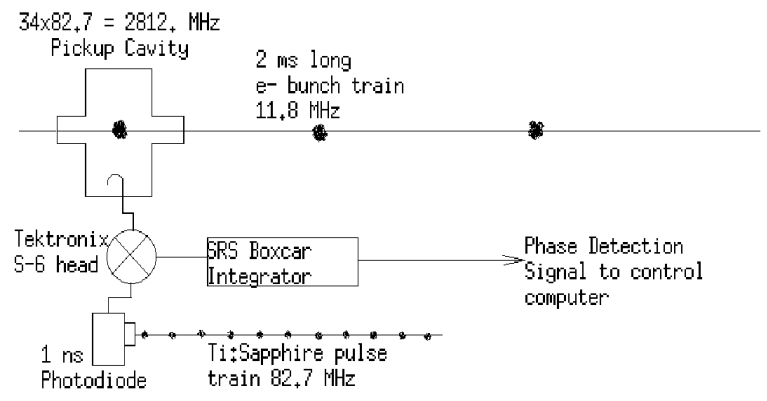

phase of the electron beam, as depicted in Fig. 2.

Figure 2: Block diagram for the fine timing detection

\subsection{Characterisation of the e-beam}

Both the fluctuations in the mean energy of the beam and its spread have been measured. The energy spread shows rather small fluctuations, however a considerable jitter in the mean energy is readily observed and has shown both short and long term oscillations. The beam has also been diagnosed near the accelerator cell and was found to show considerable jitter in the position of the beam centroid. Table 2 summarises a consistent set of beam measurements taken during a recent, parasitic run. During non-parasitic operation we have obtained a mean energy spread of $16 \mathrm{keV}$ (FWHM) or $0.05 \%$. Work is continuing on characterising and improving the e-beam quality.

Table 2: Some parasitic e-beam parameters in LEAP

\begin{tabular}{|c|l|l|}
\hline Beam & Energy spread FWHM & $37 \mathrm{keV}$ \\
\cline { 2 - 3 } Energy & r.m.s mean energy jitter & $16 \mathrm{keV}$ \\
\cline { 2 - 3 } & r.m.s. energy spread jitter & $15 \mathrm{keV}$ \\
\hline \multirow{3}{*}{$\begin{array}{c}\text { Near } \\
\text { the } \\
\text { accelerator } \\
\text { cell }\end{array}$} & horizontal position jitter & $10 \mu \mathrm{m}$ \\
\cline { 2 - 3 } & vertical position jitter & $54 \mu \mathrm{m}$ \\
\cline { 2 - 3 } & FWHM horizontal spot size & $70 \mu \mathrm{m}$ \\
\cline { 2 - 3 } & FWHM vertical spot size & $113 \mu \mathrm{m}$ \\
\cline { 2 - 3 } & Horizontal spot size jitter & $20 \%$ \\
\cline { 2 - 3 } & Vertical spot size jitter & $20 \%$ \\
\hline
\end{tabular}

\section{CALCULATIONS}

\subsection{Expected laser induced effects}

The laser beam is expected to cause a broadening in the energy spread instead of inducing an overall shift in energy. Our calculations have shown that only a very limited range of combinations of the experimental parameters can lead to a readily observable effect. The parameters that have the largest effect are the slit width, the laser pulse duration, the electron beam pulse duration and its natural energy spread.

The first step in the numerical evaluation of the laser induced energy spread profile is a diffraction calculation that finds the longitudinal electric field along the axis of propagation of the electron. The acceleration effect from two crossed gaussian beams interacting with the electron beam over a finite distance has been calculated before [7]. In order for the particle beam to enter and leave the cell an aperture of some kind has to be provided and hence partial truncation of the laser beams entering the accelerator cell and a leakage field behind the accelerator are expected. The diffraction calculation method employed here is a generalisation of a 2-D plane wave decomposition method used to find the electric field of cylindrical laser beams [8]. The expression for the longitudinal electric field at a point behind the input plane given in equation (1) of ref. [8] becomes

$$
\begin{aligned}
E_{z}(x, y, z)= & -e^{-i \omega t} \cdot \int_{-\pi / 2}^{\pi / 2} \int_{-\pi / 2}^{\pi / 2}(a(\alpha, \beta) \cdot \sin (\alpha) \cdot \\
& \left.e^{i k \cdot \sin \alpha+i k y \cdot \sin \beta} \cdot e^{i k z \sqrt{1-\sin ^{2} \alpha-\sin ^{2} \beta}}\right) \cdot d \alpha \cdot d \beta
\end{aligned}
$$

where $E_{z}$ is the longitudinal field , $a(\alpha, \beta)$ the plane wave spectrum of the input laser field and $\alpha$ and $\beta$ are the horizontal and vertical tilt angles of the plane wave with respect to the propagation axis $z$. The present calculation 
assumes that $|a(\alpha, \beta)|$ is significant only for small angles $|\alpha|,|\beta|<<1$. The effect of the slits on the electric field is very clear on Fig. 3.
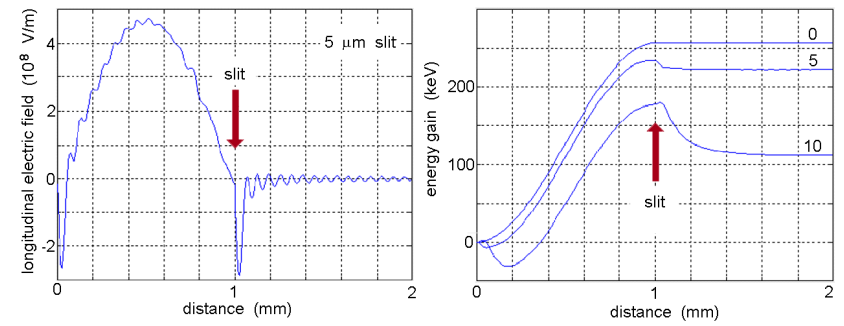

Figure 3: The longitudinal electric field and potential

The position of the exit slit is indicated with an arrow. The simulations assume a laser beam pulse of $100 \mathrm{fsec}$, having a beam waist of $65 \mu \mathrm{m}$ located at the centre of the cell with a peak intensity of $1.7 \mathrm{~J} / \mathrm{cm}^{2}$. The finite gamma due to the relatively low energy of the electron beam (32 $\mathrm{MeV}$ ) is taken into account. From Fig. 3 it can be concluded that a $10 \mu \mathrm{m}$ slit reduces the maximum attainable energy gain by one half.

\subsection{Cell length optimisation}

The optimum cell length depends on the slippage distance between the electron and the laser beam, which is a function of the energy of the of the electron beam and the angle of crossing of the laser beams. For an electron with an energy of $32 \mathrm{MeV}$ beam $(\gamma=63)$ and a laser beam at $23.44 \mathrm{mrad}$ crossing angle the slippage distance is 1.2 $\mathrm{mm}$. The energy of the beam at the SCA-FEL facility ranges between 28 and $35 \mathrm{MeV}$, which sets the optimum cell length between 700 and 800 microns, as indicated in Fig. 4.

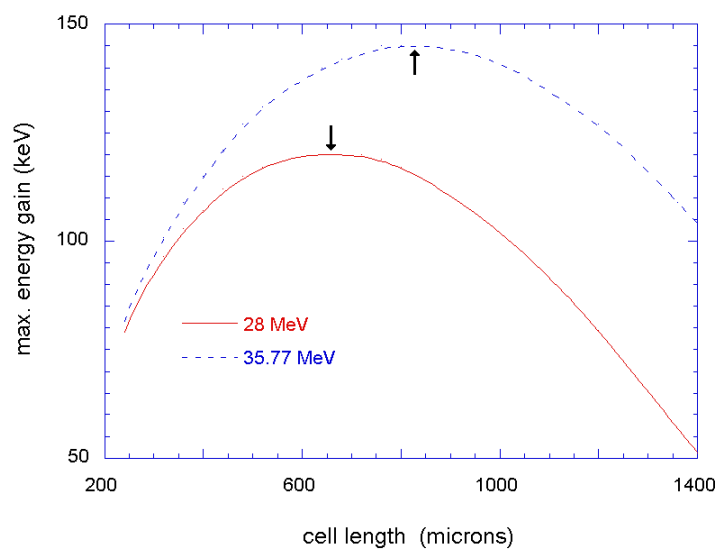

Figure 4: Dependence of the energy gain on cell length

The electron bunch length is longer than the wavelength of the laser beam by more than three orders of magnitude. Instead of experiencing a uniform energy gain due to a particular phase of the laser field the electron beam gets spread out in energy. The resulting signature in the energy spread is a function of the initial energy spread of the beam, the maximum attainable energy gain, relative pulse durations of the laser and the electron beam and the spatial overlap of the two beams.

\subsection{Calculation of the energy distribution}

After determining the maximum energy gain from the electric field calculations the numerical simulation constructs a histogram $H(E)$ of the density of electrons versus energy gain for a given laser field strength $\varepsilon$. The initial energy spread $S(E)$ is small compared to the total energy, hence it is assumed that over this small energy range the maximum gain is constant. The resulting energy spread $S^{\prime}(E, \mathcal{E})$ is

$$
S^{\prime}(E, \varepsilon)=\int S\left(E-E^{\prime}\right) \cdot H\left(E^{\prime}, \varepsilon\right) d E^{\prime}
$$

Let $N(t)$ and $\varepsilon(t)$ be the electron beam and the laser beam profile in time. The resulting energy spread $S^{\prime \prime}(E)$ becomes

$$
S^{\prime \prime}(E, \varepsilon)=\int S^{\prime}(E, \varepsilon(t)) \cdot N(t) d t
$$

Figure 5 shows the expected energy spread signatures for $5 \mu \mathrm{m}, 10 \mu \mathrm{m}$ and $20 \mu \mathrm{m}$ slits. The central peak in each case is the natural energy spread, assumed to be at its narrowest. The simulation assumes a 5 psec laser pulse. The acceleration and deceleration peaks clearly visible in the $5 \mu \mathrm{m}$ slit case are completely absent in the $20 \mu \mathrm{m}$ slit case. Further, an overall narrowing of the laser-induced energy spread can be observed as the slit is increased.
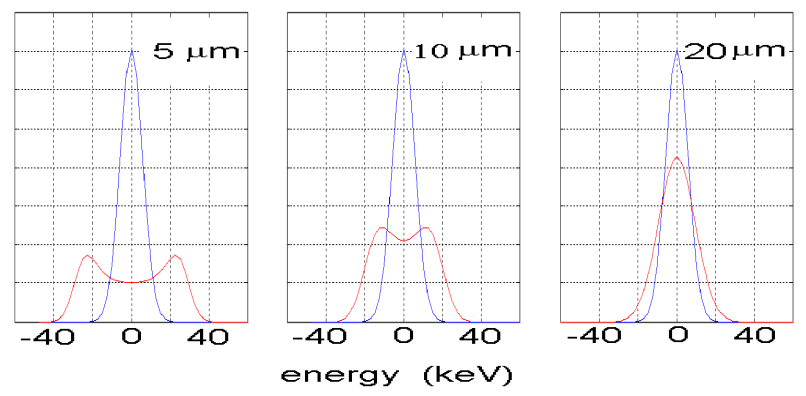

Figure 5: Energy spread signature at various slit values

Temporal overlap between the two beams also determines the energy spread signature. The pulse length of the laser available to the experiment can be set between $0.1 \mathrm{psec}$ and 30 psec. A 0.1 psec laser pulse has the highest peak field but very poor overlap with the 2 psec electron bunch and therefore a small effect on the energy spread of the electron beam. A 10 psec laser pulse illuminates the 
electron bunch almost uniformly but has a peak electric field ten times weaker than a $0.1 \mathrm{psec}$ pulse. As Fig. 6 indicates, the optimum laser pulse duration is about 1 psec, close to the electron bunch duration.
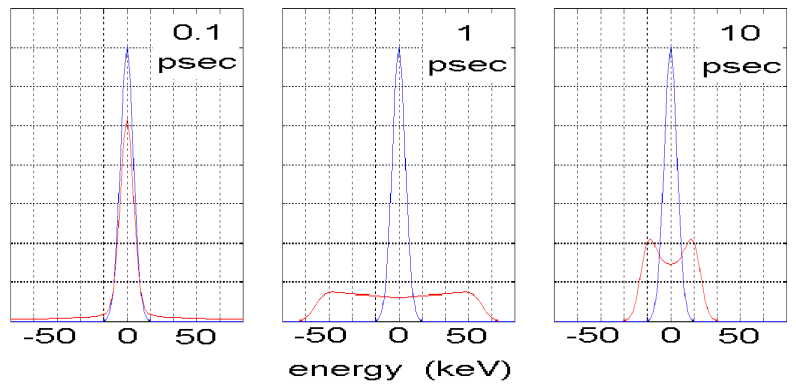

Figure 6: The effect of various laser pulse durations

\subsection{Losses from diffraction radiation}

Slits of the order of few $\mu \mathrm{m}$ in width could cause considerable energy losses on the beam due to radiative effects. An estimate of the radiation from a single electron traversing the slit was performed using a plane wave decomposition method of the field created by the particle in the slit area [9]. The energy radiated by single $32 \mathrm{MeV}$ electron traversing a $1 \mu \mathrm{m}$ infinite slit with perfect conducting boundaries was estimated to be $2 \times 10^{-3} \mathrm{eV}$. For a bunch with $10^{8}$ electrons radiating coherently the energy lost would be $200 \mathrm{keV}$ per electron. The characteristic wavelength of the radiation from the slit is

$$
\lambda \leq \frac{\text { slit width }}{\gamma}=\frac{1 \mu \mathrm{m}}{63} \sim 16 \mathrm{~nm}
$$

which corresponds to a photon energy of $\sim 77 \mathrm{eV}$. The electron beam is not optically bunched in this experiment, but is a pulse of $\sim 10^{6}$ electrons spread over $2 \mathrm{~mm}$. This corresponds to 1.3 electrons in one radian of a wave cycle. The radiation from the aperture is not coherent and hence the energy loss is negligible.

\section{CONCLUSIONS}

The numerical simulations show that in order to observe laser driven acceleration the slits of the accelerator cell may not be wider than $10 \mu \mathrm{m}$. At present we have not succeeded in achieving satisfactory transmission of the electron beam through the cell with its slits closed beyond $50 \mu \mathrm{m}$. Since the charge density of the beam is very low, no coherent radiation effects and hence no significant energy losses are expected to occur, in spite of the very narrow slit settings that the experiment requires. To obtain a better transmission through the cell improvements on the beam optics can be made. Further, a more sensitive detector can be placed at the spectrometer screen, so that even with a low transmission through the cell the experiment can be carried out. Once this obstacle is overcome we will be able to perform time scans of the laser beam and look for evidence of laser driven particle acceleration from the dielectric cell.

\section{REFERENCES}

[1] G. Mourou, et al, "Laser induced breakdown by impact ionization in $\mathrm{SiO} 2$ with pulse widths from 7 ns to $150 \mathrm{fs}$," Appl. Phys. Lett, June, 1994.

[2] B.C. Stuart, M.D. Feit, A.M. Rubenick, B.W. Shore, and M.D. Perry, Phys. Rev. Lett. 74 (12) 2248 (1995).

[3] C.M. Haarland, "Laser Electron Acceleration in Vacuum", Opt. Comm. 114, 280 (1995).

[4] Y.C. Huang, D. Zheng, W.M. Tulloch, R.L. Byer, "Proposed structure for a crossed-laser beam, $\mathrm{GeV}$ per meter gradient, vacuum electron linear accelerator", Appl. Phys. Lett. 68 (6) 753 (1996).

[5] Y.C. Huang, R.L. Byer, "A proposed high-gradient laser-driven electron accelerator using crossed cylindrical focusing" Appl. Phys. Lett. 69 (15) 2175 (1996).

[6] T. Plettner et al, Conference paper, AAC (1998).

[7] P. Sprangle, E. Esarey, J. Krall, A. Ting, Opt. Comm. 124, 69 (1996).

[8] J.A. Edinghofer and R.H. Pantell, "Energy exchange between free electrons and light in vacuum", J. Appl. Phys. 50(10) 6120 (1979).

[9] M.L. Ter-Mikaelian, "High-Energy Processes in Condensed Media, No 29, Interscience Tracts on Pysics and Astronomy, pp 377-384 